\title{
Sindrome Alcohólico Fetal como causa de Desnutrición Secundaria
}

\author{
Dra. María Meria R.$;{ }^{1}$ Srta. Carmen Alvial F.; ${ }^{2}$ Srta. Gisela Asenjo Y.: ${ }^{2}$ Srta. Erika Allendorf $A,{ }^{2}$ \\ Srta. Leila Venegas $\mathrm{Y} . i^{2}$ Dr. Hugolino Catalán $\mathrm{K}$.
}

\section{FETAL ALCOHOL SYNDROME AS A CAUSE OF SECONDARY MALNUTRITION}

\begin{abstract}
The prevalence of Jietal Aleohol Syndrome (FAS) in a nutritional center of southern Chile (Concepción) was studied during at 8 months period in 1982. 119 infants were adinitted because of undernutrition to the center, 14 (12\%,o) displayed the Jetal Alcoholic Sy ndrome, 5 were complete forms (I'AS) and 9 u'ere partial forms or Fetal Alcoholic Effects (FAE). There was practically no weight increase amone FAS, as opposed to FAE, which had a steady, even if slow, augmentation. FAS as wcll as FAE evidenced moderate mental and motor retardation, with an acceptable social adjustment and severe impairment in motor, laguage and coordination development. Infants with FAS suffered from protejn - calorie malnutrition and maternal deprivation, apart of malnutrition secondary to the sydrome. Among the heavy drinking mothers, a caloric and protein deficient diet was recorded. Paternal drinking rate was $42 \%$. On dismissal, the FAS which went back to their homes $(64 \%$ ) had worse outcome than those admitted to Foster homes or going into adoption.
\end{abstract}

El retardo del desarrollo prenatal, posnatal o de ambos es uno de los 3 rasgos clínicos principales del Sindrome Alcohólico Fetal (SAF) ${ }^{\mathbf{l}}$ y se manifiesta finalmente como déficit del estado nutricional.

El retardo del desarrollo prenatal o intrauterino se cree que puede deberse, en parte, a injuria placentaria causada por efecto tóxico direc. to del etanol o su metabolito el acetaldehido, que altera el flujo de nutrientes esenciales, como amjnoácidos, glucosa y vitaminas, durante fases críticas de la organogénesis ${ }^{2}$. También puede influir el menor consumo de alimentos por parte de las madres alcohólicas que tienden a reemplazar su ingesta de calorías por alcohol. A esto se suma la malabsorción intestinal secundaria al alcoholismo lo que provoca malnutrición matema ${ }^{3}$.

Se agrega, a la desnutrición intrauterina de los SAF, la desnutrición primaria originada, en sus hogares, por la alteración del "flujo de mutrientes" debida a que parte importante del presupuesto familiar se gasta en la adquisición de bebidas alcohólicas, resultando un menor consumo de nutrientes esenciales por los hijos. Esto se manifiesta en mayor grado cuando la madre consume gran cantidad de alcohol.

Al comprobarse en una investigación anterior sobre Alcoholisno Fetal ${ }^{4}$ que nuchos lactantes referidos a la Policlínica de Genética del Hospital G. Grant B. de Concepción, por desnut rición rebelde a tratamiento dietético, o con desarrollo psicomotor muy retardado provenian de CONIN.

1 Servicio de Pediatria - Sección! Enética, Hospital G. Grant B. Concepción.

2 CONIN de Conceprión. se decidió investigar la prevalecencia de Alcoholismo Fetal en esta institución, con el fin de contribuir al conocimiento $y$ prevención de esta causa de desnutrición secundaria. Además se deseaba conocer la evolución, el manejo apropiado; el destino y el pronóstico de los que regresaron a sus hogares o a hogares infantiles $y$ la frecuencia de alcoholismo paterno en los desnutridos primarios atendidos en CONIN.

\section{MATERIAL Y METODO}

Se revisaron las fichas clínicas y sociales de los lactantes ingresados a CONIN desde Enero a Agosto de 1982, investigando antecendes familiares de alcoholismo materno. paterno y morbilidad, con la asesoría de la Asistencia Social de la institución, como asimisno los antecedentes personales, del embarazo y el parto.

Se examinaron todos los lactantes hijos de madres bebedoras excesivas y aquellos que no subian de peso o tenian curva ponderal lenta, descartando al mismo tiempo otras enfermedades genéticas. Para el diagnóstico de SAF se empleó la clasificación del grupo de estudio de SAF con los siguientes criterios ${ }^{1}$ : Retardo del crecimiento prenatal, posnatal o ambos; signos de alteración funcional del Sistema Nervioso Central; presencia de dos de las siguientes características craneofaciales: microcefalia, microftalmia. fisuras palpebrales cortas y escaso desarrollo del surco nasolabial, labio superior fino, hipoplasia maxilar. Los hijos de madres bebedoras excesivas que presentan rasgos clínicos del síndrome pero no cumplian los requisitos de esta clasificación se denomina. ron Efectos Fetales del Alcohol (EFA) o Sindrome alcohólico fetal parcial.

Además de los rasgos enunciados pueden haber otras malformaciones de diversos órganos pe- 
ro estos no influyen en la clasificación ${ }^{\mathrm{s}}$.

La evaluación nutricional fue hecha por nutri. cionistas al ingreso, una vez al mes y al egreso de los lactantes mediante la ficha antropométrica de Sempé en uso en CONIN, que registra peso/edad, talla/edad y circunferencia de cráneo/edad.

La evaluación psicomotriz fue realizada por Educadora de Párvulos al ingreso, cada dos meses y el egreso de los lactantes, mediante la Escala de Desarrollo Psicomotor (EEDP) de Soledad Rodriguez y col.

\section{RESULTADOS}

De 119 lactantes que ingresaron en 8 meses a CONIN, $14(12 \%)$ presentaban rasgos de alcoholismo fetal: $5 \mathrm{SAF}$ completos y 9 parciales (EFA). Los 5 SAF eran mujeres, entre los EFA habian 6 hombres y 3 mujeres.

Por definición los SAF tenían mayor cantidad de rasgos clínicos que los $E F A$, lo que se puede observar en la tabla 1 , donde no aparece el peso de nacimiento o desarrollo prenatal porque se desconocían los antecedentes personales de varios lactantes por proceder de otras cuidades. Los pesos conocidos fluctuaban entre 1800 y 2600 gr. En el examen físico, además de los rasgos craneofaciales característicos, se encontraron pocas malformaciones menores y ninguna mayor, como por ejemplo cardiopatías congénitas.

Tabla 1.

Frecuencia de Rasgos Cínicos de 14 Desnutridos Secundarios a Alcoholismo Jetal Completo (SAF) y Parcial (EFA) en CONIN-Concepción

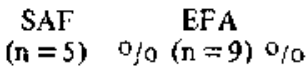

Rasgos clínicos más frecuentes

1) Retardo del desarrollo prenatal

Retardo del desarrollo

postnatal

$\begin{array}{rrrr}5 & 100 & 9 & 100 \\ 5 & 100 & 8 & 81 \\ & & & \\ 4 & 80 & 6 & 64 \\ 5 & 100 & 7 & 78 \\ 5 & 100 & 6 \pm & 64 \\ 3 & 60 & 4 \pm & 44 \\ 5 & 100 & 4 \pm & 44\end{array}$

2) Retardo del desarrollo Psicomotor

3) Alteraciones craneotaciales:

Microcefalja

Blefarofimosis

Labio superior fíno

Ausencia de sutco nasolabial 3

Hipoplasia med io facial

La curva pesojedad de los SAF fue prácticamente estacionaria, 4 lactantes con desnutriciones moderadas $y$ severas (D II y D IJI) egresaron con igual grado de desnutrición, un solo lactante mejoró en un grado su déficit nutricional. Sin embargo en general los SAF presentaron una pequeria alza ponderal debido al aporte calórico adecuado que recibían en CONIN. En la curva talla/edad se observa que también la talla estaba en rangos - 2DS $y$-3DS ( Fig. 1).

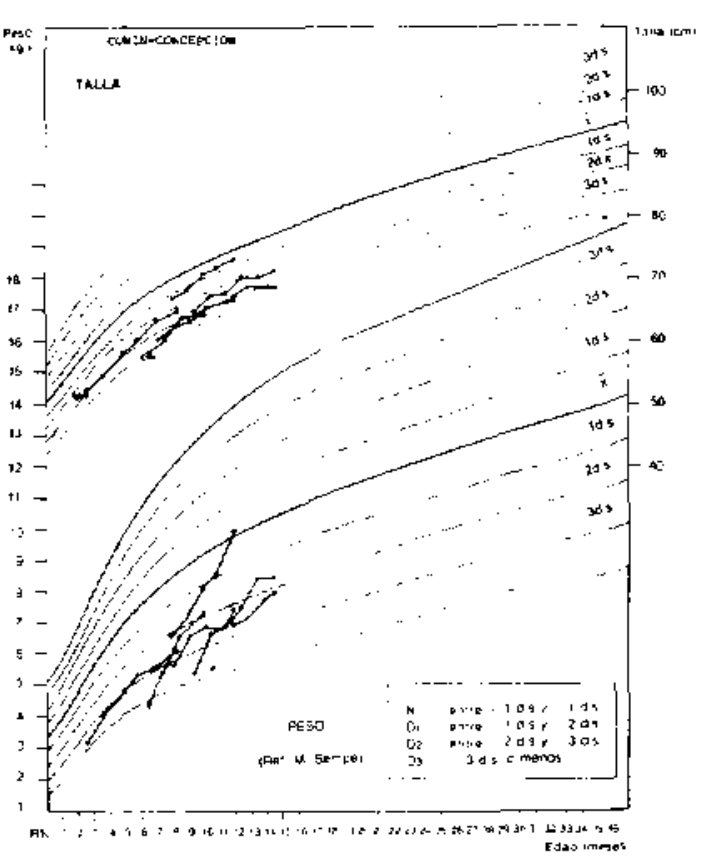

Fig. 1: Curvas de peso $y$ talla muy delicitaria en $5 \sin$. dromes alcohólicos fetales.

Comparando la curva de peso de tres de los SAF con tres desnutridos primarios de la misma edad, peso y estado nutricional se observa rápida recuperación ponderal de estos últimos y la curva plana de los SAF. No se comparó la talla de los SAF con desnutridos primarios ya que no hay grandes diferencias, no se observa ganancia de canales de crecimiento ( Fig. 2).

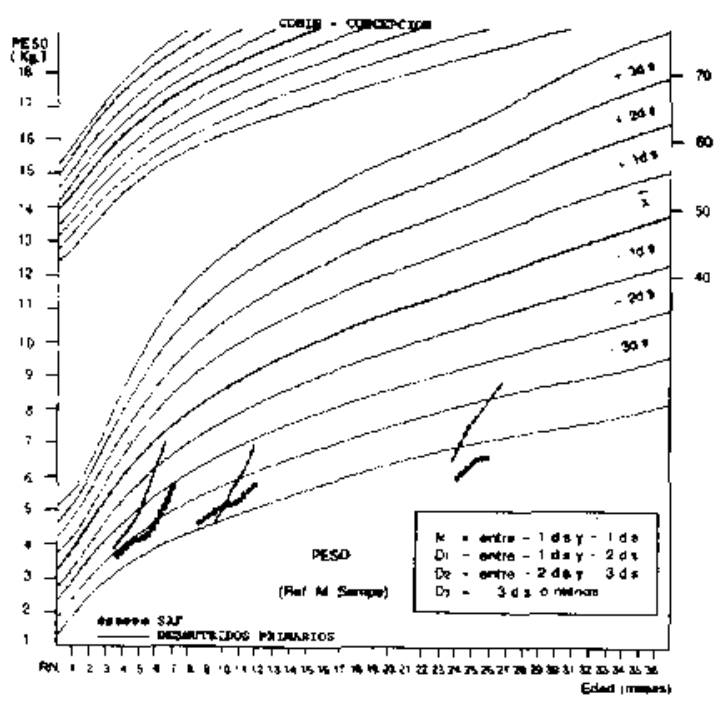

Fig. 2: Curvas ponderales muy deficitarias de 3 desnutridos secundarios a síndrome alcohólico fetal comparada con 3 desnutridos primarios con igual sexo, edad y déficit nutricional. 
La correlación perímetro de cráneo/edad en los cinco SAF está muy afectada en comparación con los desnutridos primarios; siempre se ubica bajo los 3DS ( Fig. 3).

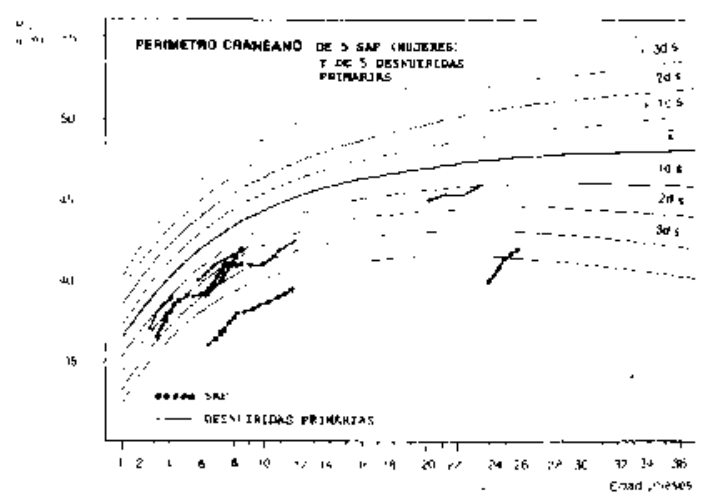

Fig. 3: Se observa gran déficit en las circunferencia. de cráneo de 5 síndromes alcohólicos fetales en comparación con $\mathbf{5}$ desnutridos primarios con igual sexo, edad $y$ déficit nutricional

En la Fig. 4 su muestran las diferencias clínicas más importantes entre un SAF y un lactante eut rófico del mismo sexo y edad.

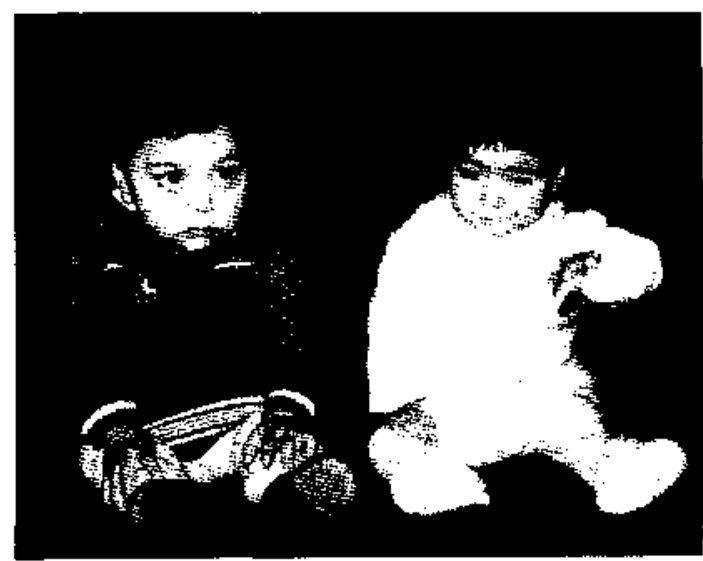

Fig 4: Dos lactantes del mismo sexo y edad: a la izquierda un eutrófico, a la derecha un portador de síndrome alcohólico fetal con desnutrición, microcefalia, blefarofimosis, labio superior fino.

En los EFA la recuperación nutricional es pa. recida a la de los desnutridos primarios pero su curva ponderal es mucho más lenta ( Fig. 5). Sin embargo también presentan microcefalia.

En cuanto al desarrollo psicomotor, los SAF ingresaron con puntajes estándar de 0,60 a 0,73 . A) egreso cuatro los habían aumentado de 0,72 a $0,86, y$ uno lo conservó en 0,73 . En la Fig. 6 se compara la evolución del desarrollo psicomotor de los cinco SAF con cinco lactantes del mismo sexo y edad, desnutridos primarios sin observar diferencias concluyentes. Al desglosar los puntajes por áreas se vió que el área motora en 4 de los 5 casos era la más afectada o con mayor retraso y el área social y de coordinación prácticamente no mostraba retraso.

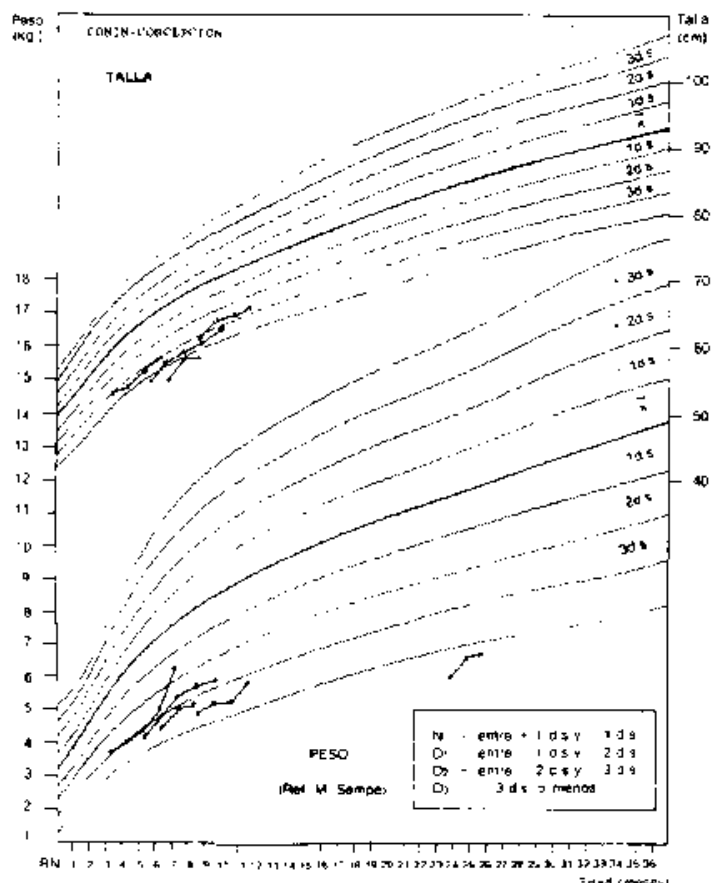

Fig 5: Curva ponderal de lactancia con efectos fetales del alcohol, parecida a la de los desnutridos primarios pero más lenta, lo mismo respec to a la talla.

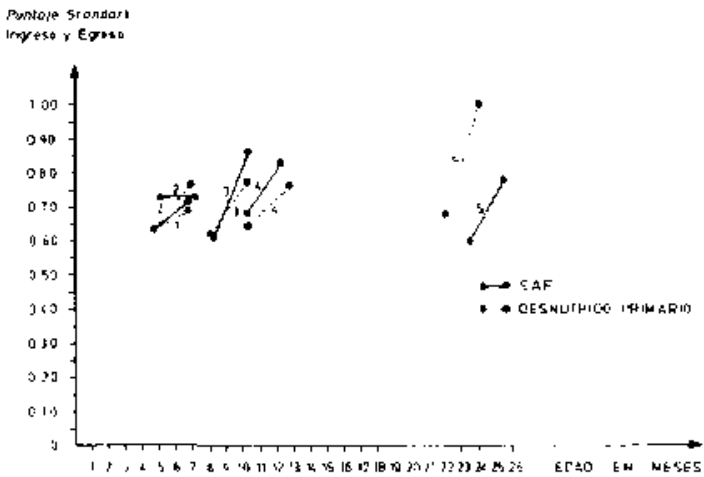

Fig. 6: Fvaluación psicomotriz de 5 lactantes con síndrome alcohólico fetal, se observa retardada con respecto a 5 desnutridos primarios de isual sexo, edad $y$ déticit nutricional. 
Los desnutridos secundarios a EFA mostraron en su desarrollo psicomotor que de 9 casos estudiados sólo 3 tuvieron un progreso importante, de puntaje estándar 0,48 a 0,79 ; de 0,67 a 0,79 y de 0,39 a 0,75 . Los restantes prácticamente no variaron. Hubo una importante coincidencia en el hecho que 8 de los 9 casos tenian muy desarrollada el área social. Tambièn existía un importante paralelismo entre dismorfogénesis y retardo psicomotor.

Durante su estadía en CONIN los lactantes con alcoholismo fetal presentaron numerosas infecciones intercurrentes especialmente bronconeumonias, diarreas agudas, otitis supuradas que originaron varias hospitalizaciones.

La mayoria de las madres refirieron ingerir aproximadamente $1 \mathrm{lt}$. de vino al día; unas pocas más de $2 \mathrm{Jt}$. de vino regularmente durante el embarazo, solamente una madre habia sido recientemente rehabilitada. En general dieron antecedentes de dietas hipocalóricas e hipoproteicas, con tendencia a preferir las calorías aportadas por el alcohol en forma de vino con harina y azúcar.

La frecuencia de ingesta exagerada de alcohol en los padres de los lactantes con SAF fue de 71\% 0 : 10 de 14 padres eran bebedores excesivos. En contraste, la frecuencia de padres bebedores excesivos en los 119 lactantes estudiados era de $42 \%$.

Al egresar de CONIN el destino de los 14 lactantes portadores de alcoholismo fetal era variable: $9(64 \%)$ regresaron con sus padres, 3 fueron a hogares infantiles, 1 a colocación familiar y otro a adopción.

Un año despuès de slk egreso de CONIN se controlaron cuatro pacientes con SAF que pudimos ubicar: Una niña de 1 ato 7 meses, había regresado con sus padres, fue visitada en su domicilio en Cocholgüe (caleta de pescadores), se encontró en pésimas condiciones, y habiendo egresado con desnutrición grado I tenja en ese momento desnutrición grạdo II, gran retardo psicomotor global, más acentuado en las áreas de lenguaje, coordinación y motora. Los $S$ hijos te. nían estigmas de Sindrome Alcohólico Fetal en diversos grados y ambos padres eran alcohólicos. El segundo niño, de 2 años 3 meses, con SAF, vi. vía en un hogar de menores, tenía déficit nutri. cional caracterizado por Pesoledad $=\mathrm{D} 3$ y Peso talla $=\mathrm{D} 2$, sin embargo, su desarrollo psicomotor tenía puntaje estándar de 0,77 (retraso leve). Otro preescolar, de 2 años 10 meses con EF A, vivia con un familiar, asistía a jardin infantil, tenía un déficit nutricional de Peso/edad = D3 y Peso/ talla $=\mathrm{D} 1$; pero su desarrollo psicomotor estaba en rangos normales.

Por último se ubicó en su domicilio, en Talca- huano un lactante de 1 año 9 meses con EF A que vivia con un familiar y asistia a un jard in infantil. La madre tenía 3 hijos más, afectados con SAF en diversos grados. El estado nutricional del lactante estaba en rangos normales, pero tenía retardo psicomotor, pues recién empezaba a caminar y tenía retardo del lenguaje.

\section{COMENTARIO}

Los lactantes con alcoholismo fetal diagnos. ticados en CONIN tenian un marcado déficit de crecimiento $y$ desarrollo secundario al Síndrome, sin embargo, éste se habia iniciado en la etapa prenatal porque los pesos de nacimiento estaban en general bajo el $\mathrm{P}_{10}$. La literatura médica refiere que los SAF presentan una desnutrición fetal precoz; pues los fetos de pocas semanas, abortados por mujeres alcohólicas, tienen menor tamafio que el correspondiente a la edad de gestación ${ }^{3}$, presentan un retardo del crecimiento pro. porcional en peso y talla, pero su circunferencia de cráneo es inferior a la correspondiente a la talla a diferencia de los fetos con desnutrición intrauterina secundaria a disfunción placentaria de los últimos meses de embarazo, en los que el crecimiento es desproporcionado, ya que se conserva el tamaño del cráneo y existe más compromiso del peso que de la talla. El retardo proporcionado de los hijos de madres alcohólicas es semejante al de los hijos de fumadoras. Sumados los dos tóxicos: alcohol y tabaco pueden provocar un severo retardo del crecimiento ${ }^{6}$.

Existen numerosas teorias sobre los mecanis. mos teratógenos del etanol. Además de actuar directamente causando injuria placentaria ${ }^{2}$. interferirja en la proliferación celular fetal, provocan do numerosas malformaciones de diversos órga nos como el sistema nervioso, los ojos, el corazón, etc., según el momento en que ocurren los episodios de intoxicación alcohólica, o los períodos gestacionales en que se producen concentraciones importantes de etanol en la sangre y los tejidos ${ }^{3}$.

Se cree que los defectos nutricionales mater. nos asociados juegan un papel importante y este antecedente se detectó en la anannesis de las ma. dres de los lactantes con alcoholismo fetal estudiados. Se sabe que la "malnurrición primaria" (debida a la baja ingestión de nutrientes en los alcohólicos), es causa e interactúa con la "malnutrición secundaria" (consecuencia de dificultades en la đigestión y absorción de nutrientes), para provocar "malnutrición terciaria" (alteración de la activación de esos nutrientes). Este conjunto de efectos "nut ricionales" potencia directamente la acción tóxica del etanol. Los órganos más afec- 
tados son el cerebro y el hígado?

Tambièn se ha informado que en los alcohólicos existe malabsorción de tiamina ${ }^{8}$, déficit de vitamina $A^{9}$, niveles séricos bajos de zinc (por dieta deficiente y pérdidas urinarias exageradas) aún sin evidencia de daño hepático ${ }^{10}$, además existen alteraciones de los aminoácidos séricos como aumentos del triptofano, fenilalanina y tirosina ${ }^{\text {"1 }}$ y déficit en la síntesis de albúmina ${ }^{12}$.

Los défjeit de vitanina $\mathrm{A}$, B y ácido fólico en la madre provocan malfomaciones diversas en el nino: microcefalia, microftalmia, defectos cardiacos, retardo mental, etc. ${ }^{3}$ Las bajas concentraciones de zinc pueden disminuir la sintesis proteica y causar retardo del crecimiento fetal general, al ta incidencia de malformaciones congénitas, aumento de la mortalidad y del efecto teratógeno del alcohol ${ }^{8}$.

En trabajos experimentales en ratas se ha demostrado que el etanol por sí solo y por inducir hipotermia, en concentraciones séricas sabre 1 $\mathrm{mg} / \mathrm{cc}$., deprime la síntesis de proteinas ${ }^{13}$. Tambiến el etanol y el acetaldehido actúan disminuyendo la sintesis de ADN especialmente del cerebro ${ }^{14}$.

En los lactantes con SAF pudimos observar un desarrollo pondoestatural estacionario. La peque. ก̃a mejoría obtenida en la curva de peso se debió seguramente al aporte alimentario adecuado suministrado en CONIN en comparación a las dietas hipocalóricas que recibían en sus hogares.

Sin embargo, al comparar las curvas de crecimiento estatural de los SAF con las de los desnutridos primarios no se encontraron mayores diferencias, siendo de recuperación lenta en ambos casos.

La curva ponderal de los EFA fue más parecida a la de los desnutridos primarios, pero aún más lenta.

Según Lemoin la recuperación del déficit pondoestatural en jos SAF es muy deficiente especialmente en la primera infancia, posteriormente en la edad escolar se recupera más rápido ${ }^{15}$.

La microcefalia era mucho más importante en los SAF que en los desnutridos primarios, esta es una de las caracteristicas más frecuentes y constantes de los SAF y EFA ${ }^{16}$.

Las alteraciones neurológicas del Sindrome se manifiestaron, en general, como retardo psicomotor moderado que se recuperó parcialmente en los $\mathrm{SAF}$ y en los EFA. Es absolutamente imposible separar el daño producido por la embriopatía alcohólica y por el ambiente, pero la existencia de este último en la forma de deprivación materna, causada por el alcoholismo y agravando los efectos orgánicos propios del sindrome, permitiría explicar la posibilidad de recuperación en condiciones ambientales más favorables.

En el estudio psicomotor de estos niños fue posible comprobar que habia áreas del desarrollo más afectadas que otras (la motriz y la del lenguaje más alteradas que la coordinación y el área social). En general los lactantes con el sindrome eran hiperkinéticos y amistosos, destacando entre el grupo de desnutridos precisamente por su sociabilidad.

Había un paralelismo constante entre los parámetros de dismorfogénesis y el retardo psicomotor, especialmente entre los SAF. En los EFA esta relación era más irregular ya que algunos de ellos tenian retardo psicomotor más importante que los $\mathrm{SAF}$.

Los lactantes afectados presentaron numerosas infecciones intercurrentes, tal como se describe en la literatura extranjera. La mayor susceptibilidad a las infecciones guarda relación con déficit inmunológicos ${ }^{17}$. También se han descrito rasgos clínicos semejantes entre el SAF y el Sindrome de Di George, cuya principal característica es la alteración del sistema inmune, sugiriéndose que en ambos actuaría un teratógeno entre la $6^{\mathrm{a}}$ y $12^{\mathrm{a}}$ semana de vida embrionaria, que interferiria con el desarrollo normal ${ }^{18}$.

El antecedente de ingestión excesiva de alcohol en las madres fue dif icil de obtener, ya que la negación del hábito forma parte de los síntomas de alcoholismo en las mujeres, que beben en forma poco notoria, en dosis moderada, pero frecuentemente; razón por la que no es fácil establecer una relación entre cantidad ingerida de alcohol y grado de alcoholismo fetal.

En general la cantidad de alcohol ingerida por las madres fue muy inferior a la referida en la literatura extranjera ${ }^{19}$, por esta razón probablemente no se observan malformaciones congénitas más severas, como cardiopatías congénitas, que aparecen con elevada frecuencia en otras series clínicas ${ }^{20-21}$. Se podría deducir que en estas madres la dosis de teratógeno era relativamente menor, pero a su yez potenciada por la malnutri. ción materna. Al respecto existe un estudio realizado en la Universidad de Concepción en autopsias de cirróticos etílicos, en que a las mujeres corresponde el $36 \%$ de los casos, siendo en ellos la malnutrición un factor constante ${ }^{22}$.

Un elevado indice de ingesta excesiva de alcohol paterno en el grupo de lactantes con alcoholismo fetal, sugiere que el pronóstico de éstos es mucho peor por tener ambos padres alcohólicos.

En cuanto al destino de los lactantes con alcoholismo fetal al alta de CONIN la mayoría $(640 \%)$ regresó con sus padres teniendo ellos e] peor pronóstico en cuanto a nutrición y desarro. 
llo psicomotor; los restantes fueron a hogares infantiles o adopción, siendo el pronóstico mejor.

Sin embargo, existe la impresión de que la defjciencia de crecimiento y desarrollo persistentes de los SAF no es consecuencia del ambiente postnatal, ni se debe a un déficit de hormona de crecimiento. La ausencia de crecimiento rápido aún con alimentación y atención óptimas, indica la desfavorable situación biológica en que se encuentran estos niños, refractaria a la nomalización de los factores exógenos tanto ambientales como alimentarios. También está comprometido el desarrollo cerebral como lo atestigua la persistente microcefalia, los trastomos psicomotores $y$ los del comportamiento que presenten estos enfermos ${ }^{24}$.

Se pudo observar en el segu imiento de algunos de los lactantes con alcoholismo fetal, un año đespués de egresar, que persistía un déficit nutricional importante tanto en los lactantes con SAF de vuelta a sus hogares como en aquellos enviados a hogares infantiles; pero el déficit fue más acentuado en los que estaban con sus padres. Además el desarrollo psicomotor global era mucho mejor, especialmente el desarrollo motor y del lenguaje, en los niños que estaban en hogares infantiles o con otros familiares que los que permanecian con sus padres.

Creemos que es importante conocer las carac. terísticas del SAF para poder diagnosticarlo y prevenirlo, educar a la población y aconsejar la abstención del alcohol durante el embarazo y la lactancia. Se ha comprobado que el alcohol pasa a la leche $y$ disminuye su producción ${ }^{16}$. Esta información debe darse regularmente a las personas que se controlan en el Servicio de Salud porque en las anamnesis frecuentemente se obtuvo el antecedente de ingestión de alcohol durante el embarazo y la lactancia; pues tradicionalmente se trasmite la idea de que el alcohol sería beneficioso para el feto y posteriomente para el lactante.

\section{RESUMEN}

Se investiga la prevalencia de Alcoholismo Fe. tal en CONIN de Concepción. De 119 lactantes ingresados durante 8 meses en 1982, $14(12 \%)$ tenían Sindrome Alcohólico Fetal: 5 completos (SAF) y 9 parciales, Efectos Fetales de Alcohol (EFA).

La recuperación ponderal de los SAF fue prácticamente nula, en los EFA la curva de peso mostró ascenso lento.

Se observó que el retardo psicomotor de los SAF y EFA era moderado, con conservación del área social y mayor deterioro en las áreas motora, lenguaje y coordinación.
Los lactantes con SAF presentaban desnutrición primaria y deprivación materna además de la desnutrición secundaria al Síndrome.

En las madres bebedoras excesivas además se observó una dieta hipocalórica e hipoproteica.

La frecuencia de padres bebedores excesivos era de $42 \%$.

Al alta los SAF que fueron a sus hogares $(64 \% \%)$ tenian peor pronóstico que los que fueron a hogares, colocaciones familiares o adopción.

\section{REFERENCIAS}

${ }^{1}$ Rosett, H.L.; Weinier, L.: "Strategies for prevention of Fetal Alcohol Effects". Obstetric and Ginecology, 57: 1, 1981.

2 Fisher, S.: Atkinson, $M_{\text {.: }}$ "E,thanol-Associated Selec. tive Fetal Malnutrition: A contributing Factor in the FAS", Alcoholism, 6: 197, 1982.

${ }^{3}$ Hinkers, H.I: "The influences of alcohol on the fetus", J. Petinat. Med. 6: 3, 1978.

"Mena, M.; Selman, E.: "Sindrome Fetal Alcohólico". Rev, Chil. Ped. 53: 127, 1982.

5 Clarren, $S, K$ : : "Recognition of J.AS". J.A.M.A. 45: 2436, 1981.

"Sokol, R.: Miller, Sh.; Reed, G.: "Alcohol Abuse during pregnancy: An epidemjologic study". Alcoholisin 4,: $135,1980$.

7 tieber, $C h$. . "Interactions of alcohol and nutrition" Alcoholism. 7: 2, 1983.

${ }^{8}$ Hoyumpo, A.M.: "Alcohol and Thiamine Metabo. lism". Alcoholism, 7: 11, 1983.

9 Leo, M.A.; Lieber, Ch.: "Interaction of Ethenol with vitamin A". Alcoholism, 7: 15, 1983

10 Mc Clain, C.J.: "Zinc Deficiency in the Alcoholic: A Reyjew". 7: 5, 1983.

11 Shat, S. Lieber, Ch, "Plasma Amino Acids in the Alcoholic: Nutritional aspect. Alcoholism, 7: 22, 1983.

12 Rothschild, M.A.; Oratz, M.: "Effects of Nutrition and Alcohoi on Albumin Sy nthesis". Alcoholism ?: 28,1983 .

13 Henderson, G.; Hayumpa, $A_{1}$ : "Etfect of Ethanolinduced hypothermia on protein synthesis in pregnant and fetal rats". Alcoholism 4: 165, 1980.

14 Dreosti, J.: Ballard, J, Belling, B.: "The effects of Ethanol and Acetaldehyde on D.N.A, development in the rat". Alcoholisn 5:357, 1981.

15 Lemoine, P.; Haronsseau, H.L.: "Les cnfants de parents alcoholiques. Anomalies obscrvées a propos dd 127 cas". Quest Med. 25: 476, 1968.

16 Surgeon General Advisary on Alcohol and Pregnancy. Clin, Pediat. 21: 16, 1982.

17 Johnson, Sh. Knight, R.: "Inmune deticience in Fetal Alcohol Syndrome". Pediatrics Research, 15: 908,1981 .

18 Ammanh, A,J.: Warg, D.W.: "The Di George Syndrome and the fetal Alcohol Syndrome". Am. J. Dis. Child., 136: 906, 1982.

19 Josu, S.; Fuchs, M.: "Long Term Follow up of three siblings witl Fetal Alcohol Syndrome". Alcoholism, 5: $523,1981$.

20 Hanson, J." Woolf, P.: "Cardiovascular maltormations in the Fetal Alcohol Syndrome. Experienoe with 41 patients". J.A.M.A. 235: 1458, 1976.

21 Steeg. $C_{\text {. }}$ Woolf, $P_{*}$ : "Cardiovascular malformations 